\title{
ARTICLES \\ STOCHASTIC INTEREST RATES AND AUTOREGRESSIVE INTEGRATED MOVING AVERAGE PROCESSES
}

\author{
By JAN DHAEne \\ Instituut voor Actuariële Wetenschappen, K.U.Leuven, Belgium
}

\begin{abstract}
A practical method is developed for computing moments of insurance functions when interest rates are assumed to follow an autoregressive integrated moving average process.
\end{abstract}

\section{KEYWORDS}

$\operatorname{ARIMA}(p, d, q)$-processes; stochastic interest rates; moments of insurance functions.

\section{INTRODUCTION}

In most of the insurance literature the theory of life contingencies is developed in a deterministic way. This means that mortality happens according to an a priori known mortality table and that the interest rate is assumed to have a constant value. Nevertheless, the traditional theory of life contingencies implicitly deals with the stochastic nature of mortality and interest rates in that conservative assumptions are taken.

A first step forward was to consider the time until decrement as a random variable, while the interest rate was assumed to be constant. This approach is followed in BowERs et al. (1987). This (as one could call) "semi-stochastic" approach contains the traditional theory in that most actuarial functions can be considered as the expected values of certain stochastic functions.

It is only since about 1970 that there has been interest in actuarial models which consider both the time until death and the investment rate of return as random variables.

Boyle (1976) includes the stochastic nature of interest rates in assuming that the force of interest is generated by a white noise series, that is forces of interest in the successive years are normally distributed and uncorrelated.

In the approach of Pollard (1971) the force of interest in a year is related to the force of interest in the preceding years by using an autoregressive process of order two.

Panjer and Bellhouse (1980) and Bellhouse and Panjer (1981) develop a general theory including continuous and discrete models. The theory is further worked out for unconditional and conditional autoregressive processes of order one and two.

ASTIN BULLETIN, Vol. 19, No. 2 
GiaccotTo (1986) develops an algorithm for evaluating present value functions when interest rates are assumed to follow an $\operatorname{ARIMA}(p, 0, q)$ or an $\operatorname{ARIMA}(p, 1, q)$ process.

The goal of this study is to state a methodology for computing in an efficient manner present value functions when the force of interest evolves according to an autoregressive integrated moving average process of order $(p, d, q)$. As will be seen, the method developed here will require less computing time than Giaccotto's method for autoregressive integrated moving average processes of order $(p, 0, q)$ or $(p, 1, q)$.

It should be remarked that we assume that mortality and interest rates posses a certain stochastic nature and that only accidental fluctuations in this mortality and interest rates are considered. Other fluctuations due to mortality improvement, underwriting practice, the choice of a wrong interest model, investment strategy and so on are not considered here.

\section{GENERAL THEORY}

The theory developed in this section is mainly based on the work of PANJER and Bellhouse (1980) and Bellhouse and Panjer (1981).

Let $D_{t}$ be the stochastic variable denoting the discounted value of one dollar payable in $t$ years $(t=0,1,2, \ldots)$. The stochastic variable $X_{t}$ defined by

$$
D_{t}=\exp \left(-X_{t}\right) \quad t=0,1,2, \ldots
$$

can be interpreted as the force of interest over the first $t$ years.

If $\delta_{i}$ is the force of interest in the $i$-th year $(i=1,2, \ldots)$, then

$$
X_{0}=0
$$

$$
X_{t}=\sum_{i=1}^{t} \delta_{i} \quad t=1,2, \ldots
$$

It is assumed that $X_{t}$ is normally distributed with mean $\mu(t)$ and variancecovariance function $a(t, s)$. The variance of $X_{t}$ is equal to $a(t, t)$ and is denoted by $\sigma^{2}(t)$.

It is immediately seen that $E\left[D_{t}^{k}\right]$ and $E\left[D_{t}^{k} D_{s}^{l}\right]$ are the moment generating functions of the normal distributed variables $k X_{t}$ and $\left(k X_{t}+l X_{s}\right)$ calculated for the value $(-1)$. So one finds that

$$
E\left[D_{t}^{k}\right]=\exp \left[-k \mu(t)+\frac{k^{2}}{2} \sigma^{2}(t)\right] \quad t, k \geq 1
$$

and

$$
\begin{aligned}
& E\left[D_{t}^{k} D_{s}^{l}\right]=\exp \left[-k \mu(t)-l \mu(s)+\frac{k^{2}}{2} \sigma^{2}(t)+\right. \\
& \left.+\frac{l^{2}}{2} \sigma^{2}(s)+k l a(t, s)\right] \quad t, s, k, l \geq 1
\end{aligned}
$$


Panjer and Bellhouse (1980) proved that when the $X_{t}$ are normally distributed, the moments of and the correlation coefficients between interest, annuity and insurance functions depend upon $E\left[D_{t}^{k}\right]$ and $E\left[D_{t}^{k} D_{s}^{l}\right]$. For a whole life term insurance, for instance, the moments of the stochastic variable $A_{x}$ are given by

$$
E\left[A_{x}^{k}\right]=\sum_{t=1}^{\infty} t-1 \mid q_{x} E\left[D_{t}^{k}\right]
$$

The second moment for the life annuity $\underset{\sim}{a}$ is given by

$$
E\left[a_{x}^{2}\right]=\sum_{t=1}^{\infty} t \mid q_{x} \sum_{r=1}^{t} \sum_{s=1}^{t} E\left[D_{r} D_{s}\right]
$$

Given a model for the yearly forces of interest $\delta_{t}$, the problem is to find $\mu(t)$, $\sigma^{2}(t)$ and $\alpha(t, s)$ for $t, s \geq 1$.

\section{AUTOREGRESSIVE INTEGRATED MOVING AVERAGE PROCESSES}

Assume that the stochastic model governing future forces of interest $\delta_{t}$ $(t=1,2, \ldots)$ belongs to the class of $\operatorname{ARIMA}(p, d, q)$-processes. Then $\delta_{t}$ is generated by the stochastic difference equation

$$
\begin{aligned}
\nabla^{d} \delta_{t}= & \mu+b_{1}\left(\nabla^{d} \delta_{t-1}-\mu\right)+b_{2}\left(\nabla^{d} \delta_{t-2}-\mu\right)+\ldots+b_{p}\left(\nabla^{d} \delta_{t-p}-\mu\right) \\
& +\xi_{t}-c_{1} \xi_{t-1}-c_{2} \xi_{t-2} \ldots-c_{q} \xi_{t-q}
\end{aligned}
$$

where $\nabla^{d}$ stand for the $d$-th backward difference operator:

$$
\begin{gathered}
\nabla^{1} \delta_{t} \equiv \nabla \delta_{t}=\delta_{t}-\delta_{t-1} \\
\nabla^{d} \delta_{t}=\nabla\left(\nabla^{d-1} \delta_{t}\right) \quad d=2,3, \ldots
\end{gathered}
$$

By convention we set $\nabla^{0} \delta_{t}=\delta_{t}$. Further $\xi_{i}$ is a normal white noise series with mean zero and variance $\sigma^{2}$. Equation (7) can also be written as

$$
\nabla^{d} \delta_{t}=a+b_{1} \nabla^{d} \delta_{t-1}+\ldots+b_{p} \nabla^{d} \delta_{t-p}+\xi_{t}-c_{1} \xi_{t-1}-\ldots-c_{q} \xi_{t-q}
$$

with a given by

$$
a=\mu\left(1-\sum_{i=1}^{p} b_{i}\right)
$$

Equation (7) indicates that the process describing $\delta_{t}$ will not necessary be stationary. This means that the force of interest $\delta_{t}$ will not necessary have a constant unconditional mean, variance and autocovariance with any $\delta_{t-k}$ for $t \neq k$. The $d$-th difference of $\delta_{t}$ however follows a stationary autoregressive moving average process. This means that the series describing the interest rate exhibits homogeneity in the sense that, apart from local level, or perhaps local level and trend, one part of the series behaves much like any other part. 
In what follows it will implicitly be assumed that the past $(p+d)$ forces of interest $\delta_{0}, \delta_{-1}, \ldots, \delta_{1-p-d}$ and the past $q$ random disturbances $\xi_{0}, \ldots, \xi_{1-q}$ are known. Means, variances and covariances will always be considered as conditional on $\delta_{0}, \delta_{-1}, \ldots, \delta_{1-p-d}, \xi_{0}, \xi_{-1}, \ldots, \xi_{1-q}$. Remark that if $\delta_{t}$ follows an $\operatorname{ARIMA}(p, d, q)$-process then the $X_{t}$ given by (2) are normally distributed so that the theory of section 2 can be used.

The variable $Y_{t}$ is defined as

$$
Y_{t}=\delta_{1-p-d}+\delta_{2-p-d}+\ldots+\delta_{t} \quad t \geq 1-p-d
$$

Further we set

$$
Y_{-p-d}=0
$$

It follows immediately that

$$
\delta_{t}=Y_{t}-Y_{t-1} \quad t \geq 1-p-d
$$

So if $\delta_{t}$ follows an $\operatorname{ARIMA}(p, d, q)$-process given by (10) with $\delta_{0}, \ldots, \delta_{1-p-d}, \xi_{0}, \ldots, \xi_{1-q}$ known then $Y_{t}$ follows an ARIMA $(p, d+1, q)$ process given by

$$
\nabla^{d+1} Y_{t}=a+b_{1} \nabla^{d+1} Y_{t-1}+\ldots+b_{p} \nabla^{d+1} Y_{t-\mathrm{p}}+\xi_{t}-c_{1} \xi_{t-1}-\ldots-c_{q} \xi_{t-q}
$$

with $Y_{-p-d}, Y_{1-p-d}, \ldots, Y_{0}$ and $\xi_{0}, \xi_{-1}, \ldots, \xi_{1-q}$ known.

Now it is easy to see that the ARIMA $(p, d+1, q)$-process describing $Y_{t}$ can be written as an ARIMA $(l, 0, q)$-process with $l=p+d+1$ :

$$
Y_{t}=a+\phi_{1} Y_{t-1}+\ldots+\phi_{t} Y_{t-1}+\xi_{t}-c_{1} \xi_{t-1}-\ldots-c_{q} \xi_{t-q}
$$

with $\phi_{1}, \phi_{2}, \ldots, \phi_{l}$ suitable functions of $b_{1}, \ldots, b_{p}$.

\section{Examples}

(1) If $\delta_{t}$ follows an ARIMA $(p, 0, q)$-process then

$$
\delta_{t}=\mu+b_{1}\left(\delta_{t-1}-\mu\right)+\ldots+b_{p}\left(\delta_{t-p}-\mu\right)+\xi_{t}-c_{1} \xi_{t-1}-\ldots-c_{q} \xi_{t-q}
$$

$Y_{t}$ can then be written as an ARIMA $(p+1,0, q)$-process given by (18) $Y_{t}=a+\phi_{1} Y_{t-1}+\ldots+\phi_{p+1} Y_{t-p-1}+\xi_{t}-c_{1} \xi_{t-1}-\ldots-c_{q} \xi_{t-q}$ with

$$
a=\mu\left(1-\sum_{i=1}^{p} b_{i}\right)
$$

and

$$
\phi_{i}=b_{i}-b_{i-1} \quad i=1, \ldots, p+1
$$

with $b_{0}=-1$ and $b_{p+1}=0$ 
(2) If $\delta_{t}$ follows an ARIMA $(p, 1, q)$-process then

(21) $\nabla \delta_{t}=\mu+b_{1}\left(\nabla \delta_{t-1}-\mu\right)+\ldots+b_{p}\left(\nabla \delta_{t-p}-\mu\right)+\xi_{t}-c_{1} \xi_{t-1}-\ldots-c_{q} \xi_{t-q}$ $Y_{t}$ can then be written as an ARIMA $(p+2,0, q)$-process given by

(22) $Y_{t}=a+\phi_{1} Y_{t-1}+\ldots+\phi_{p+2} Y_{t-p-2}+\xi_{t}-c_{1} \xi_{t-1}-\ldots-c_{q} \xi_{t-q} \quad t \geq 1$

with

$$
a=\mu\left(1-\sum_{i=1}^{p} b_{i}\right)
$$

and

$$
\phi_{i}=b_{i}-2 b_{i-1}+b_{i-2} \quad i=1, \ldots, p+2
$$

with $b_{-1}=b_{p+1}=b_{p+2}=0$ and $b_{0}=-1$

In the next lemma we derive an expression for the $Y_{t}$ in terms of known values plus a function of future error terms $\xi_{t}$.

\section{Lemma 1}

Assume that $Y_{t}$ moves according to an $\operatorname{ARIMA}(l, 0, q)$-process given by (16) and with $Y_{0}, Y_{-1}, \ldots, Y_{1-l}$ and $\xi_{0}, \xi_{-1}, \ldots, \xi_{1-q}$ known. The $Y_{t}$ can be written as

$$
\begin{aligned}
Y_{t} & =\sum_{i=1}^{l} Y_{i-l} \sum_{j=\max (0, i-t)}^{i-1} \phi_{l-j} a_{j-i+t} \\
& -\sum_{i=1}^{q} \xi_{i-q} \sum_{j=\max (0, i-t)}^{i-1} c_{q-j} a_{j-i+t}+a \sum_{i=0}^{t-1} a_{i}+\sum_{i=0}^{t-1} \beta_{i} \xi_{t-i} \quad t \geq 1
\end{aligned}
$$

where the coefficients $\alpha_{i}$ and $\beta_{i}$ are given by

$$
\begin{gathered}
a_{0}=1, \quad \beta_{0}=1 \\
a_{i}=\sum_{j=1}^{\min (i, l)} \phi_{j} a_{i-j} \quad i \geq 1 \\
\beta_{i}=a_{i}-\sum_{j=1}^{\min (i, q)} c_{j} a_{i-j} \quad i \geq 1
\end{gathered}
$$

\section{Proof}

For arbitrary constants $a_{i}(i=0,1, \ldots, t-1)$ we find for $t \geq 1$ $\sum_{i=0}^{t-1} a_{i} Y_{t-i}=\sum_{j=1}^{l} \phi_{j} \sum_{i=j}^{t+j-1} a_{i-j} Y_{t-i}-\sum_{j=1}^{q} c_{j} \sum_{i=j}^{t+j-1} a_{i-j} \xi_{t-i}+\sum_{i=0}^{t-1}\left(a+\xi_{t-i}\right) a_{i}$ 
By interchanging the order of summation in the second member of this equation and by using the $\alpha_{i}$ and $\beta_{i}$ defined in (26), (27) and (28) we find

$$
\begin{aligned}
Y_{t}= & \sum_{i=t}^{t+l-1} Y_{t-i} \sum_{j=i-t+1}^{\min (i, \eta)} \phi_{j} a_{i-j}-\sum_{i=t}^{t+q-1} \xi_{t-i} \sum_{j=i-t+1}^{\min (i, q)} c_{j} a_{i-j} \\
& +\mathrm{a} \sum_{i=0}^{t-1} a_{i}+\sum_{i=0}^{t-1} \beta_{i} \xi_{t-i}
\end{aligned}
$$

After some straightforward calculation (25) is obtained.

Remark that the first, the second and the thirth term in the right member of (25) are constants while the fourth term is stochastic.

In the following theorem expressions are derived for computing $\mu(t), \sigma^{2}(t)$ and $a(t, s)$.

\section{Theorem 1}

If $Y_{t}$ follows an ARIMA $(l, 0, q)$-process given by $(16)$ then $\mu(t), \sigma^{2}(t)$ and $a(t, s)$ can be computed by

$$
\mu(t)=a-Y_{0}\left(1-\sum_{i=1}^{l} \phi_{i}\right)+\sum_{i=1}^{l} \phi_{i} \mu(t-i)-\sum_{i=1}^{q} c_{i} \eta(t-i) \quad t \geq 1
$$

where $\mu(0)=0$ and $\mu(-i)=-\left(\delta_{0}+\ldots+\delta_{1-i}\right) \quad i=1, \ldots, l-1$

and $\eta(i)=\left\{\begin{array}{cc}\xi_{i} & i \leq 0 \\ 0 & i>0\end{array}\right.$

$$
\sigma^{2}(t)=\sigma^{2} \sum_{i=0}^{t-1} \beta_{i}^{2}=\sigma^{2}(t-1)+\beta_{t-1}^{2} \quad t \geq 1
$$

with $\sigma^{2}(0)=0$ and the $\beta_{i}$ defined in (26), (27) and (28).

$$
a(t, s)=\sigma^{2} \sum_{i=1}^{s} \beta_{t-i} \beta_{s-i} \quad t>s \geq 1
$$

\section{Proof}

From (2), (12) and (16) we obtain

$$
X_{t}=-Y_{0}+a+\phi_{1} Y_{t-1}+\ldots+\phi_{1} Y_{t-1}+\xi_{t}-c_{1} \xi_{t-1}-\ldots-c_{q} \xi_{t-q} \quad t \geq 1
$$

Taking the expected value of both members gives (29). (30) and (31) follow immediately from (25).

The results obtained in lemma 1 and theorem 1 become much simpler if $Y_{t}$ follows an ARIMA $(l, 0,0)$-process. The expressions to compute $\mu(t), \sigma^{2}(t)$ and $a(t, s)$ for this case are stated in the following theorem. 


\section{Theorem 2}

If $Y_{l}$ follows an ARIMA $(l, 0,0)$-process given by (16) with $c_{1}=c_{2}=\ldots=c_{q}=0$ then $\mu(t), \sigma^{2}(t)$ and $\alpha(t, s)$ can be computed by

$$
\mu(t)=a-Y_{0}\left(1-\sum_{i=1}^{l} \phi_{i}\right)+\sum_{i=1}^{l} \phi_{i} \mu(t-i) \quad t \geq 1
$$

where $\mu(0)=0$ and $\mu(-i)=-\left(\delta_{0}+\ldots \delta_{1-i}\right) \quad i=1, \ldots, l-1$

$$
\sigma^{2}(t)=\sigma^{2} \sum_{i=0}^{t-1} a_{i}^{2}
$$

with $\sigma^{2}(0)=0$ and the $\alpha_{i}$ defined in (26) and (27)

$$
a(t, s)=\sigma^{2} \sum_{i=1}^{s} a_{t-i} a_{s-i} \quad t>s \geq 1
$$

The proof follows immediately from theorem 1 by deleting the terms in $c_{i}(i=1, \ldots, q)$.

\section{REMARKS}

The method described by GiaccotTo (1986) for ARIMA $(p, 0, q)$ - and $\operatorname{ARIMA}(p, 1, q)$-processes requires for the computation of $\sigma^{2}(t)$ values of $x_{i}(t)$ and $y_{i}(t)(i=1, \ldots, t)$, which can be computed recursively but that depend on $t$. In the method developed here for computing $\sigma^{2}(t)$, the algorithm is written so that the $a_{i}$ - and $\beta_{i}$-values are independent of $t$.

We remark from theorem 1 and 2 that $\sigma^{2}(t)$ and $a(t, s)$ are independent of the past forces of interest $\delta_{0}, \delta_{-1}, \ldots, \delta_{1-1}$. So it follows that when the same interest rate model is used from year to year with only the past $l$ forces of interest and the past $q$ disturbances changing, the $\sigma^{2}(t)$ and $a(t, s)$ remain the same. Only the $\mu(t)$ will have to be recomputed every year.

\section{EXAMPLE}

To use our results the following procedure should be followed:

1) Choose an ARIMA $(p, d, q)$ interest rate model and estimate the parameters involved. (see e.g. Box and JENKINS (1970)).

2) Write $Y_{t}$ as an ARIMA $(p+d+1,0, q)$-process.

3) Compute the $\alpha_{i}$ 's and the $\beta_{i}$ 's.

4) Compute $\mu(t), \sigma^{2}(t), a(t, s)$.

5) Compute the moments of actuarial functions.

To illustrate the procedure assume that we have the following model for the interest rate:

$$
\delta_{t}=0.08+0.6\left(\delta_{t-1}-0.08\right)-0.3\left(\delta_{t-2}-0.08\right)+\xi_{t} \quad t \geq 1
$$


where $\xi_{t}$ is a white noise series with variance 0.0016 and $\delta_{0}=0.06$ and $\delta_{-1}=0.07$.

Using (18), (19) and (20) $Y_{t}$ can be written as

$$
Y_{t}=0.056+1.6 Y_{t-1}-0.9 Y_{t-2}+0.3 Y_{t-3}+\xi_{t} \quad t \geq 1
$$

The $a_{t}, \mu(t), \sigma^{2}(t)$ and $a(t, s)$ can then be computed by using theorem 2 and formula (26) and (27).

In table $1 a_{t}, \mu(t), \sigma^{2}(t), E\left[D_{t}\right]$ and $\operatorname{Var}\left[D_{t}\right]$ are given for $t=0,1, \ldots, 5$. In the last column the discounted value of $1 \$$ payable in $t$ years computed with a constant force of interest equal to the unconditional expected value of $\delta_{t}$ is given. In the example described here the stochastic approach leads to higher single premiums. This fact could be expected by observing $\delta_{0}$ and $\delta_{-1}$.

TABLE 1

MEAN AND VARIANCE OF A PAYMENT OF 1 DUE IN $t$ Years

\begin{tabular}{ccccccc}
\hline \hline$t$ & $a_{t}$ & $\mu(t)$ & $\sigma^{2}(t)$ & $E\left[D_{t}\right]$ & $\operatorname{Var}\left[D_{t}\right]$ & $\exp (-0.08 t)$ \\
\hline 0 & 1 & 0 & 0 & 1 & 0 & 1 \\
1 & 1.6000 & 0.0710 & 0.0016 & 0.9322 & 0.0014 & 0.9231 \\
2 & 1.6600 & 0.1516 & 0.0057 & 0.8618 & 0.0042 & 0.8521 \\
3 & 1.5160 & 0.2347 & 0.0101 & 0.7948 & 0.0064 & 0.7866 \\
4 & 1.4116 & 0.3163 & 0.0138 & 0.7339 & 0.0075 & 0.7261 \\
5 & & 0.3964 & 0.0170 & 0.6784 & 0.0080 & 0.6703 \\
\hline
\end{tabular}

\section{ACKNOWLEDGEMENT}

The author wishes to thank the anonymous referees for their helpful comments.

\section{REFERENCES}

Bellhouse, D. R. and PANJer, H. H. (1981) Stochastic modelling of interest rates with applications to life contingencies - part II. Journal of Risk and Insurance, vol. XLVII no. 4, 628-637.

Bowers, N. L., Gerber, H.U., Hickman, J.C., Jones, D. A. and Nesbit, C.J. (1987) Actuarial Mathematics, Society of Actuaries.

Box, G. E. P. and Jenkins, G. M. (1970) Time Series Analysis. Holden-Day, San Francisco.

BoYLE, P.P. (1976) Rates of return as random variables. Journal of Risk and Insurance, vol. XLIII no. 4, 693-713.

Giaccotтo, C. (1986) Stochastic modelling of interest rates: actuarial vs. equilibrium approach. Journal of Risk and Insurance, vol. LIII no. 3, 435-453.

Panjer, H. H. and Bellhouse, D. R. (1980) Stochastic modelling of interest rates with applications to life contingencies. Journal of Risk and Insurance, vol. XLVII no. 1, 91 110.

Pollard, J.H. (1971) On fluctuating interest rates. Bulletin van de Koninklijke Vereniging van Belgische Actuarissen nr. 66, 68-97.

\section{JAN DhaENE}

Instituut voor Actuariële Wetenschappen, K.U.Leuven, Dekenstraat 2, 3000 Leuven, Belgium. 